\title{
Severe insulin resistance in long-term acute leukaemia survivors: lesson learned from a clinical case and review of the literature
}

\author{
BILAL BASHIR, MOULINATH BANERJEE
}

Key words: insulin resistance, metabolic syndrome, leukaemia, chemotherapy, radiotherapy, thiazolidinediones, metformin

\begin{abstract}
With the improvement of haematopoietic stem cell transplantation (HSCT) and radiotherapy, the population of cancer survivors is increasing and therefore increasing the number of patients living with late metabolic complications. We describe a case of a childhood acute lymphoblastic leukaemia survivor who developed insulin resistance 10 years after HSCT and total body radiation requiring a high dose of insulin (>1,500 IU). Using insulin-sensitising agents metformin and thiazolidinediones improved the control and reduced the insulin requirement - eventually stopping insulin. We describe for the first time the phenomenon of reverse diurnal variation in insulin sensitivity based on the clinical picture alone, which has not previously been described in the literature. We have reviewed the plausible mechanisms of developing insulin resistance, reverse diurnal variation and the role of thiazolidinediones in reducing lipotoxicity and adipocyte differentiation resulting in improved insulin sensitivity in such cases.
\end{abstract}

\section{Introduction}

The prevalence of developing type 2 diabetes and metabolic syndrome is $3 \%$ with autologous stem cell transplantation and $8-41 \%$ in allogeneic stem cell transplantation. ${ }^{1}$ Recognition of late development of metabolic syndrome is important with an increasing number of childhood cancer survivors.

\section{Case report}

A 26-year-old Caucasian female presented 18 months after receiving a diagnosis of type 2 diabetes mellitus. She had been diagnosed with acute lymphoblastic leukaemia (ALL) at the age of 10 years in 1986. She was treated with UKALL-10 (schedule

Department of Diabetes, Endocrinology and Specialist Weight Management, Bolton NHS Foundation Trust, Bolton, UK

Address for correspondence: Dr Moulinath Banerjee Department of Diabetes, Endocrinology and Specialist Weight Management, Bolton NHS Foundation Trust, Bolton BL4 OJR, UK E-mail: mbanerjee@nhs.net

Br J Diabetes 2021;21:255-259

https://doi.org/10.15277/bjd.2021.326
D including 18 Gy cranial irradiation) followed by total body irradiation (TBI) and bone marrow allograft after pre-conditioning in 1990 leading to long-term remission. This was followed by growth hormone deficiency, premature ovarian failure which was treated with hormone replacement therapy, bilateral ovarian masses requiring salpingo-oophorectomy at 16 years, bilateral cataracts at 19 years and breast cancer at 41 years resulting in left mastectomy and adjuvant chemotherapy. She did not have a family history of diabetes in her first-degree relatives. She developed diabetes in 2000 at the age of 24 years, and had her first consultation with our team in 2002 when she was being treated with pre-mixed twice daily insulin with a total daily dose of 32 units. Her self-monitored blood glucose readings averaged 16-20 mmol/L and her $\mathrm{HbA}_{1 \mathrm{c}}$ was $12 \%$ (IFCC $108 \mathrm{mmol} / \mathrm{mol}$ ). Her insulin dose and regimen were altered over the next few years and she was transitioned from pre-mixed twice daily insulin to a multiple daily injection basal-bolus regime with the dose gradually escalated up to 1,500 units/day over the next 10 years, yet glycaemic control remained suboptimal with $\mathrm{HbA}_{1 \mathrm{c}}$ at 12 years from the diagnosis (2012) $10.4 \%(90 \mathrm{mmol} / \mathrm{mol})$. She was commenced on metformin, pioglitazone and rosiglitazone during this period on more than one occasion; however, compliance with oral hypoglycaemic agents remained suboptimal due to patient-reported gastrointestinal side effects.

She developed severe acanthosis nigricans in 2005 at the neck and in the axilla and, at that point, marked diurnal variation in insulin sensitivity was observed. Diurnal variation in insulin sensitivity became more apparent while she was on continuous subcutaneous insulin infusion during 2007-2018, when she was advised to suspend the insulin infusion overnight, yet maintaining capillary blood glucose 4-8 mmol/L. However, her capillary blood glucose rose to 16-20 mmol/L during the day time even during carbohydrate-free days. She was not on any prescribed or over-the-counter drugs to account for this remarkable variation in glycaemia/insulin resistance.

Eighteen years from diagnosis she was tried again on slowrelease metformin and pioglitazone (15 mg once daily). She was compliant with these drugs and did not report any significant side effects. With the introduction of these agents we observed a significant improvement in her glycaemic control and a marked reduction in her insulin requirement. She was gradually weaned off insulin therapy and stopped later in the year 2018.

Her current therapeutic regimen includes pioglitazone $30 \mathrm{mg}$ once daily and metformin SR $1000 \mathrm{mg}$ twice daily with $\mathrm{HbA}_{1 c}$ of 
Figure 1. Serial $\mathrm{HbA}_{1 \mathrm{c}}$ over time

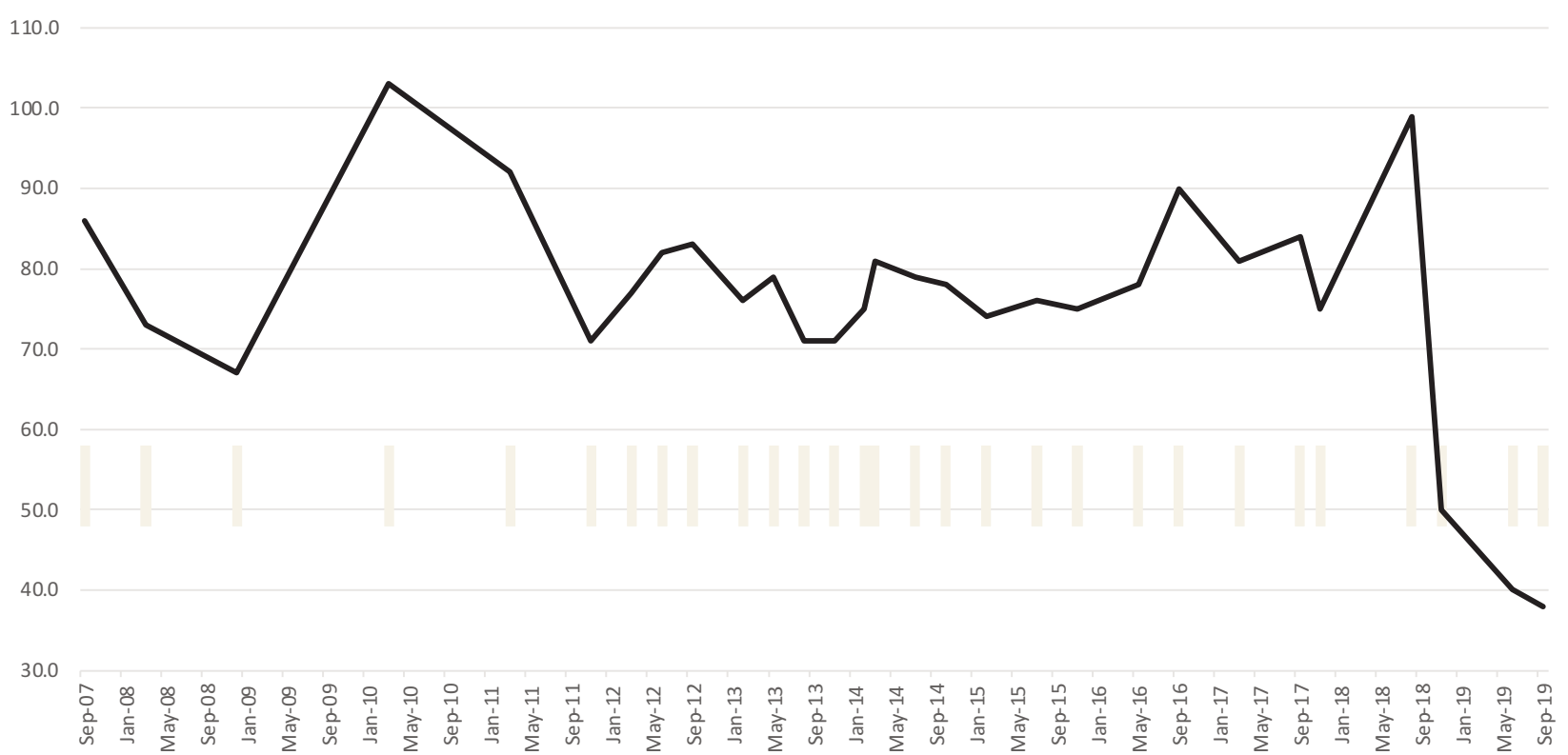

$6 \%$ (IFCC $39 \mathrm{mmol} / \mathrm{mol}$ ), average self-monitored blood glucose $4-10 \mathrm{mmol} / \mathrm{L}$, a steady body mass index of $24.3 \mathrm{~kg} / \mathrm{m}^{2}\left(28.1 \mathrm{~kg} / \mathrm{m}^{2}\right.$ in 2000) and a normal renal and lipid profile. Despite improvement in her glycaemic profile, we did not observe a significant improvement in acanthosis nigricans. Her serial $\mathrm{HbA}_{1 \mathrm{c}}$ is shown in Figure 1 and the journey of escalation and de-escalation of treatment is shown in Table 1.

\section{Discussion}

Our unique case depicts severe insulin resistance manifested by severe acanthosis nigricans and suboptimal glycaemic control despite being on extremely high doses of insulin with a significant response to insulin-sensitising agents. She also demonstrated a reversal of diurnal insulin resistance that has not been reported before.

The genesis of insulin resistance and diabetes after HSCT/TBI is poorly understood. Proposed mechanisms involve increased fat mass, reduced lean body mass, anthropometric changes from abnormal fat distribution leading to a phenotypic picture of sarcopenic obesity and lipodystrophy, altered dynamics of adipokines secondary to abnormal fat distribution, chronic inflammatory milieu and accelerated cellular aging process. ${ }^{2}$

The exact mechanism of abnormal fat dynamics in these individuals is not known. Cranial irradiation leads to hypothalamic-pituitary dysfunction and leptin resistance which reduces lean body mass and increases fat mass and insulin resistance. ${ }^{3,4}$ After irradiation, depletion of the adipocyte pool, changes in its morphology and the inability of adipose tissue to store lipids upon reaching its maximal capacity lead to ectopic fat deposition in the muscles, liver and pancreas. ${ }^{5}$ This hypothesis is supported by Lei et a/ who found a lower body mass index and higher intramuscular to total fat ratio in HSCT+TBI recipients compared with those receiving chemotherapy alone or an obese otherwise healthy adult group (Figure 2). ${ }^{6}$
Severe insulin resistance and its metabolic consequences are reversed to some extent by thiazolidinediones via their action on adipocytes, as observed in our case and seen in patients with lipodystrophies. ${ }^{7}$ Thiazolidinediones induce differentiation of preadipocytes leading to the production of smaller more insulin-sensitive adipocytes and apoptosis of larger insulin-resistant visceral adipocytes, reduce circulating triglycerides, decrease the expression of resistin, interleukin 6 and tumour necrosis factor $\alpha$ and promote adiponectin. This change in the metabolomic and proteomic profile is associated with an improvement in insulin sensitivity. Thiazolidinediones also enhance the expression of GLUT1 and GLUT4 in skeletal muscles and adipocytes increasing their glucose uptake, which contributes to a reduction in the glucose load and thereby improves insulin sensitivity. ${ }^{8}$

Another striking feature observed in our case was severe acanthosis nigricans, which has been considered as a surrogate marker for laboratory measurement of insulin resistance. Neck acanthosis nigricans has been described as having a sensitivity of $96 \%$ for insulin resistance. ${ }^{9}$ This is in contrast to localised acanthosis nigricans, which can develop in response to cutaneous injection of insulin and is reversible upon cessation of insulin or changing the site of injection. ${ }^{10}$ Neck and axilla acanthosis nigricans in our patient indeed suggest extreme insulin resistance rather than high-dose insulin as a cause of acanthosis nigricans. Phiske et al postulated increased adiposity and an imbalance in adipokine secretion (decreased serum adiponectin, increased serum resistin and decreased adiponectin gene expression) as a possible mechanism for the development of insulin resistance in these patients and hence acanthosis nigricans. ${ }^{9}$ Our case demonstrated improved insulin sensitivity in response to thiazolidinedione which is in keeping with the probable mechanism proposed by Kodawaki et a/ where thiazolidinedione is believed to increase the circulating levels of adiponectin by altering the mor- 
Table 1 Summary of escalation and de-escalation of treatment over time

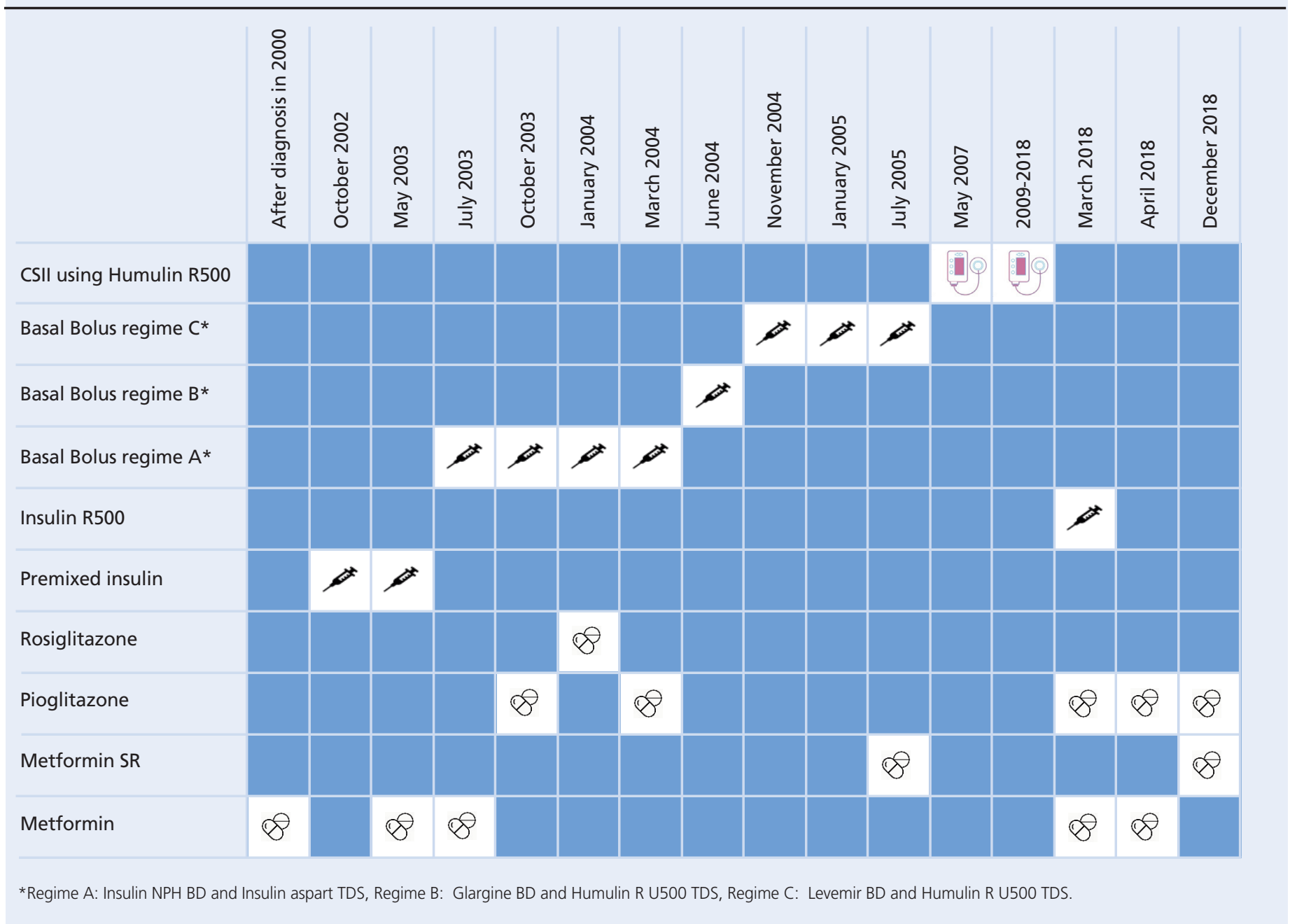

phology and differentiation of adipocytes and upregulating the adiponectin receptors AdipoR1 and AdipoR2. ${ }^{11}$ Although acanthosis nigricans is a sensitive surrogate marker for insulin resistance, improvement in acanthosis nigricans was not observed in our case, consistent with reported literature.

The literature on the insulin-sensitising effect of metformin in lipodystrophy phenotypes is limited and confined to HIV-associated lipodystrophies. A meta-analysis of six trials has demonstrated significant improvement in insulin sensitivity in this cohort of patients. ${ }^{12}$ There is a paucity of data on the effect of metformin on lipodystrophy phenotype secondary to HSCT/TBI; however, the beneficial effect of the insulin-sensitising action of metformin in this scenario cannot be overlooked, as demonstrated in our patient where we used a combination of metformin and thiazolidinedione and a case reported by Wedrychowicz et a/ where the introduction of only metformin halved the insulin requirement in a patient with diabetes post-HSCT/TBI. ${ }^{13}$

The diurnal variation in insulin sensitivity, which has been described in the literature, shows increased insulin resistance during the dark phase (ie, night-time during sleep). The proposed mechanisms to explain this phenomenon include alteration in free fatty acid availability, clock genes influencing insulin sensitivity at different times of day, diurnal rhythm in sympathetic activity and expression of intrinsic circadian rhythm in adipose tissue. ${ }^{14,15}$ Reverse diurnal variation has been described in animal models after reversing the light-dark cycle and has been attributed to diurnal variation in growth hormone and nocturnal surges of growth hormone, ${ }^{16}$ but not in humans. Ding et al have demonstrated differential expression of nuclear receptors in the suprachiasmatic nucleus which controls the diurnal rhythm of insulin sensitivity. ${ }^{17}$ Similarly, the diurnal variation in free fatty acid availability governed by previous meals and diurnal expression of the PDK4 gene responsible for the availability of free fatty acids govern insulin sensitivity. ${ }^{18}$ It is not known if cytotoxic chemotherapy and radiotherapy, which work by inducing DNA, can disrupt the intrinsic circadian rhythm leading to insulin resistance. However, altered expression of these clock genes has been shown to induce changes in the glycometabolic profile. ${ }^{17,19}$ We believe that growth hormone deficiency and the lack of nocturnal growth hormone surges alone are insufficient to explain insulin sensitivity in our case.

An increasing number of cases of childhood leukaemia are surviving with improvement in oncological management. They pose a 
Figure 2. Figure 2 Pathophysiology of insulin resistance after bone marrow transplantation (BMT), chemotherapy and radiotherapy

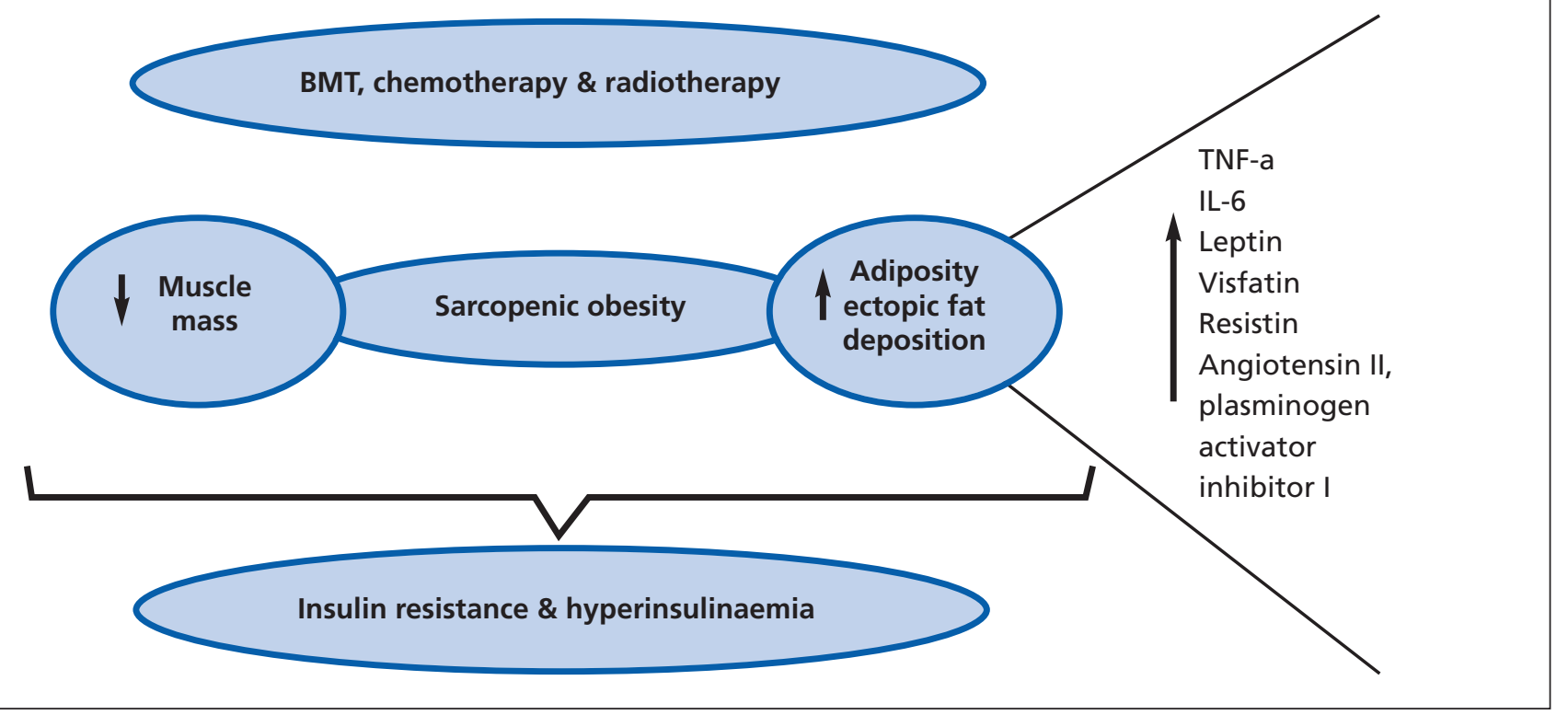

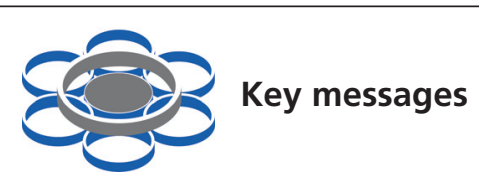

- Development of metabolic syndrome is a known late complication of haematopoietic stem cell transplantation/ total body irradiation in long-term cancer survivors

- Extreme insulin resistance is conferred due to redistribution of fat mass and reduction in lean body mass

- Literature on the role of pharmacological agents in reversing extreme insulin resistance in these cases is limited to case reports

- Reversal of extreme insulin resistance is possible with thiazolidinediones and metformin

- We have demonstrated and reviewed the potential mechanisms of extreme diurnal variation in insulin resistance that have not previously been reported

- Clinicians are encountering an increasing number of childhood cancer survivors with metabolic syndrome and diabetes. The standard escalation regimen to achieve adequate glycaemic control in these cases might not work

- Early initiation of insulin sensitisers rather than insulin secretagogues or insulin itself is important in such cases. The role of newer antidiabetic regimens (ie, sodiumglucose cotransporter-2 inhibitors and glucagon-like peptide-1 agonists) has not been studied in this subgroup and provides a potential avenue for further research

significant therapeutic challenge. In these patients, sensitivity would need to be preserved with insulin sensitisers rather than insulin secretagogues or insulin itself. The role of glycosuric agents (sodiumglucose cotransporter-2 inhibitors) in this group of patients is not yet known. Further studies are required to address late metabolic complications and optimum pharmacological management in this subset of patients.

\section{Study limitations}

This study has the following limitations: (1) acute lymphoblastic leukaemia treatment, induction, maintenance chemotherapy and radiotherapy were carried out at a different centre and we were unable to retrieve the complete details from that centre; (2) we did not measure C-peptide and hence baseline insulin reserve at presentation; and (3) we have not quantified the insulin resistance by formal testing; however, the development of severe acanthosis nigricans suggests marked insulin resistance.

\section{Conflict of interest None.}

Funding None.

Ethical approval Written informed consent was obtained from the patient.

Author contributions BB: literature review and writing the original draft. $\mathrm{MB}$ : conception of the idea, management of the case, reviewing and editing the draft, supervision.

\section{References}

1. Inamoto $Y$, Lee SJ. Late effects of blood and marrow transplantation. Haematologica 2017;102(4):614-25. https://doi.org/10.3324/haematol. 2016.150250

2. Turcotte LM, Yingst A, Verneris MR. Metabolic syndrome after hematopoietic cell transplantation: at the intersection of treatment toxicity and immune dysfunction. Biol Blood Marrow Transplant 2016; 22(7):1159-66. https://doi.org/10.1016/j.bbmt.2016.03.016

3. Link K, Moëll C, Garwicz S, et al. Growth hormone deficiency predicts cardiovascular risk in young adults treated for acute lymphoblastic leukemia in childhood. J Clin Endocrinol Metab 2004;89(10):5003-12. https://doi.org/10.1210/jc.2004-0126

4. Considine RV, Sinha MK, Heiman ML, et al. Serum immunoreactive-leptin concentrations in normal-weight and obese humans. $N$ Engl J Med 1996;334(5):292-5. https://doi.org/10.1056/NEJM199602013340503 
5. Poglio S, Galvani S, Bour S, et al. Adipose tissue sensitivity to radiation exposure. Am J Pathol 2009;174(1):44-53. https://doi.org/10.2353/ajpath.2009.080505

6. Wei C, Thyagiarajan MS, Hunt LP, Shield JPH, Stevens MCG, Crowne EC. Reduced insulin sensitivity in childhood survivors of haematopoietic stem cell transplantation is associated with lipodystropic and sarcopenic phenotypes. Pediatr Blood Cancer 2015;62(11):1992-9. https://doi.org/ $10.1002 / p b c .25601$

7. Sutinen J. The effects of thiazolidinediones on metabolic complications and lipodystrophy in hiv-infected patients. PPAR Res 2009;2009: 373524. https://doi.org/10.1155/2009/373524

8. Thangavel N, Al Bratty M, Akhtar Javed S, Ahsan W, Alhazmi HA. Targeting peroxisome proliferator-activated receptors using thiazolidinediones: strategy for design of novel antidiabetic drugs. Int J Med Chem 2017:2017:1069718. https://doi.org/10.1155/2017/1069718

9. Phiske M. An approach to acanthosis nigricans. Indian Dermatol Online J 2014;5(3):239-49. https://doi.org/10.4103/2229-5178.137765

10. Yahagi $E$, Mabuchi $T$, Nuruki $H$, et al. A case of exogenous insulinderived acanthosis nigricans caused by insulin injections. Tokai J Exp Clin Med 2014;39(1):5-9.

11. Kadowaki T, Yamauchi T, Kubota N, Hara K, Ueki K, Tobe K. Adiponectin and adiponectin receptors in insulin resistance, diabetes, and the metabolic syndrome. J Clin Invest 2006;116(7):1784-92. https://doi.org/ $10.1172 / \mathrm{JCl} 29126$

12. Sheth SH, Larson RJ. The efficacy and safety of insulin-sensitizing drugs in HIV-associated lipodystrophy syndrome: a meta-analysis of randomized trials. BMC Infect Dis 2010;10(1):183. https://doi.org/10.1186/ 1471-2334-10-183
13. Wędrychowicz A, Ciechanowska M, Stelmach M, Starzyk J. Diabetes mellitus after allogeneic hematopoietic stem cell transplantation. Horm Res Paediatr 2013;79(1):44-50. https://doi.org/10.1159/000345547

14. Yoshino J, Almeda-Valdes P, Patterson BW, et al. Diurnal variation in insulin sensitivity of glucose metabolism is associated with diurnal variations in whole-body and cellular fatty acid metabolism in metabolically normal women. J Clin Endocrinol Metab 2014;99(9):E1666-70. https://doi.org/10.1210/jc.2014-1579

15. Carrasco-Benso MP, Rivero-Gutierrez B, Lopez-Minguez J, et al. Human adipose tissue expresses intrinsic circadian rhythm in insulin sensitivity. FASEB J 2016;30(9):3117-23. https://doi.org/10.1096/fj.201600269RR

16. Shih K-C, Liu L-Y, Kwok C-F, et al. Effect of reversing dark-light cycles on normal diurnal variation and related metabolic disturbance in rats. Chin J Physio/ 2007;50(2):69-76. Available from: http://europepmc.org/ abstract/MED/17608144

17. Ding G, Li X, Hou X, et al. REV-ERB in GABAergic neurons controls diurnal hepatic insulin sensitivity. Nature 2021;592(7856):763-7. https://doi.org/10.1038/s41586-021-03358-w

18. Yamaguchi S, Moseley AC, Almeda-Valdes $P$, et al. Diurnal variation in PDK4 expression is associated with plasma free fatty acid availability in people. J Clin Endocrinol Metab 2018;103(3):1068-76. https://doi.org/ 10.1210/jc.2017-02230

19. Stenvers DJ, Scheer FAJL, Schrauwen P, la Fleur SE, Kalsbeek A. Circadian clocks and insulin resistance. Nat Rev Endocrinol 2019;15(2):75-89. https://doi.org/10.1038/s41574-018-0122-1 